\title{
A joint moment projection method and maximum entropy approach for simulation of soot formation and oxidation in diesel engines
}

\author{
Shaohua $\mathrm{Wu}^{\mathrm{a}}$, Chung Ting Lao ${ }^{\mathrm{b}}$, Jethro Akroyd ${ }^{\mathrm{b}}$, Sebastian Mosbach ${ }^{\mathrm{b}}$, \\ Wenming Yang ${ }^{\mathrm{a}}$, Markus Kraft*b,c \\ ${ }^{a}$ Department of Mechanical Engineering, National University of Singapore, \\ Engineering Block EA, Engineering Drive 1, Singapore, 117576 \\ ${ }^{b}$ Department of Chemical Engineering and Biotechnology, University of Cambridge, \\ West Cambridge Site, Philippa Fawcett Drive, Cambridge, CB3 OAS United Kingdom \\ ${ }^{c}$ School of Chemical and Biomedical Engineering, Nanyang Technological University, \\ 62 Nanyang Drive, Singapore, 637459 \\ corresponding author* \\ E-mail:mk306@cam.ac.uk
}

\begin{abstract}
A joint moment projection method and maximum entropy approach for treating the soot population balance equations is developed and presented in this work. The moment projection method is used to solve the population balance equations and generate moments that are supplied to the maximum entropy approach as a post-processing technique to reconstruct the soot particle size distribution. The particle size range required by the maximum entropy for particle size distribution reconstruction is determined based on the weighted particles generated in the moment projection method. The performance of the joint approach is first evaluated by solving a set of simplfied population balance equations on MatLab, then it is implemented into a Stochastic Reactor Model engine code to simulate the formation and oxidation of soot particles in a single-cylinder direct injection diesel engine. Results suggest
\end{abstract}


that the joint approach has the advantages of ease of implementation, high accuracy and low computational cost. It enables a detailed analysis on the soot formation and oxidation processes in diesel engines. Complete information on the soot particle size distribution can be provided with little CPU cost induced.

Keywords: soot, moment projection method, maximum entropy, particle size distribution, diesel engine

\section{Introduction}

Diesel engine is of critical importance to transportation and power generation owing to its high thermal efficiency and low fuel consumption [1-5]. It is expected to remain as a major power source in the next few decades. However, the existence of locally high equivalence ratios together with high temperature zones inside the engine cylinder lead to the formation of substantial amounts of soot particles, which are found to be major causes of several environmental and health problems [6-12]. Therefore, the worldwide regulations on soot emissions from diesel engines are becoming more and more strigient, which limits the viability of diesel engines. In order to reduce the soot emissions and achieve cleaner combustion, modelling soot formation and oxidation in diesel engines is seen to have increasing importance.

The literatures [13-15] on simulations of soot formation and oxidation in diesel engines are largely based on simplified empirical soot models, most of which belong to the two-step model $[16,17]$ and its variants $[18,19]$. The soot formation is modeled to be linked directly to the fuel concentrations using lumped reactions with usually very high activation energy [18]. The oxidation 
of soot particles is often described using the Nagle and Strickland-Constable oxidation model [20]. These empirical soot models have advantages of ease of implementation and low computational costs but having the drawbacks of a poor representation of the physical and chemical sooting processes. Since the detailed soot particle dynamics is neglected, limited soot formation and oxidation details can be provided and these models often suffer from a narrow range of applicability.

The formation of soot is a rather sophisticated process including both gas phase chemistry and particle dynamics depending on a wide range of parameters [21-27]. Starting from the formation of polycyclic aromatic hydrocarbons (PAHs) due to fuel pyrolysis and oxidation, these PAHs collide with each other to form the first primary soot particles [28]. Once the particles are formed, their population may evolve through the collision and sticking which occur amongst soot particles. They may also grow or decrease in size due to surface reactions with gaseous molecules. Up to nowadays, numerous soot models have been proposed to describe the detailed soot formation and oxidation processes such as the spherical model [29, 30], surface volume model [31] and primary particle model [32]. Different soot models focus on different aspects of the soot formation process and give rise to different complexity. Nevertheless, all these soot models can be described by using the so-called population balance equations (PBEs) [33] which are, in mathematics, a series of partial differential equations that are very complex to solve. Kraft and co-workers [34-36] have adopted a stochastic method to treat the soot PBEs in internal combustion engines. The stochastic method [37-39] is intuitive and accurate. It allows for the direct calculation of the soot particle 
size distribution (PSD) characterizing particles by multiple properties. The simulations have been proven to converge to the deterministic solution of the PBEs. However, the coupling between the soot models and the gas-phase chemistry within the framework of the stochastic method is not trivial. The simulations are prohibitively expensive and limited to single molecules [40]. The method of moments (MOM) is a promising approach for simulating soot formation in diesel engines owing to its low computational cost and ease of implementation. Instead of solving the particle PBEs directly, it transforms the PBEs into moment equations and solves for just the first few lower order moments, thus reducing the computational cost significantly. However, the application of MOM for the simulations of soot formation in diesel engines has been limited in two aspects. On the one hand, the soot moment equations are usually unclosed. The realistic collision kernels lead to the presence of fractional-order moments in the moment equations which are not directly solved for and have to be properly estimated [41-45]. An even more challenging closure problem arises from the soot oxidation process where the number of the smallest soot particles is required to evaluate the soot boundary flux term [46-48]. On the other hand, the low computational cost of MOM comes at the expense of limited information on the soot particles that can be provided [49]. Since the detailed soot PSD has been transformed into moments, only integral quantities such as the total soot number and mean particle size can be generated by MOM.

Up to date, numerous moment methods have been proposed for the handling of the moment closure problems. Among them, the moment projection method (MPM) $[50,51]$ is attractive for its robustness and high accuracy. 
In MPM, the PSD is approximated by a set of weighted particles with one particle size fixed at the smallest size to evaluate the boundary flux term due to oxidation. The performance of MPM has been tested extensively and it has shown to be very accurate for all particle processes including oxidation. However, like the traditional MOM, MPM can only be used to generate integral particle quantities. To overcome this limitation, several continuous kernel function based moment methods have been proposed [47, 49, 52]. In the finite-size domain complete set of trial functions method of moments (FCMOM) [53], a series of Legendre polynomials are adopted to estimate the unclosed moment source terms and approximate the PSD. Since limited numbers of polynomials can be determined, FCMOM cannot guarantee the positivity of the approximated PSD. This problem is avoided in the extended quadrature method of moments (EQMOM) [54] by representing the PSD with a set of non-negative kernel density functions such as gamma function or beta function. EQMOM has shown to be able to achieve high accuracy in terms of the reconstructed PSD. However, it requires prior information on the shape of the PSD to select a suitable kernel function and the resulting moment equations are usually so complex that it is not trivial to be directly implemented for soot simulation in diesel engines. Instead of solving directly for the PSD, a possible approach belongs to one of those moment reversion algorithms such as the Maximum Entropy (ME) technique [55, 56]. In ME, the PSD is reconstructed with a series of non-negative exponential polynomials based on just a few lower-order moments. Given a proper particle size range and corresponding moments, the reconstructed PSD by ME can be of high accuracy. 
The purpose of this paper is to present a new approach for simulating soot formation in diesel engines. A joint MPM-ME approach is developed which is able to provide a comprehensive study on the soot particle dynamics and at the same time retain the advantages of ease of implementation, low computational cost and high accuracy. The paper is organized as follows. First, the engine and soot models are introduced together with a brief description of the moment projection method. Then the detailed algorithm of the ME technique is described. In section 3, the performance of the MPM-ME approach is evaluated with a set of simplified particle PBEs. In section 4, this new approach is implemented into a Stochastic Reactor Model (SRM) engine code to simulate the soot formation and oxidation processes inside a singlecylinder direct injection diesel engine which is operated under three different conditions. Finally some key conclusions are summarized.

\section{Numerical methodology}

\subsection{Engine model}

The Probability Density Function (PDF) based Stochastic Reactor Model (SRM) engine code is a spatially zero-dimensional model applicable for physical and chemical processes including Internal Combustion (IC) engines [35]. It has been successfully applied for combustion simulation in a number of IC engines including Direct Injection Spark Ignition (DISI) engines [34], Homogeneous Charge Compression Ignition (HCCI) engine [35] and Direct Injection Compression Ignition engine (DICI) [57]. The code possesses sub-models for turbulent mixing, fuel spray, flame propagation and heat transfer. Within the framework of SRM, the scalar variables such as species concentrations, 
temperatures as well as the soot moments are represented by a so-called "stochastic particle". The engine in-cylinder charge can then be split into an ensemble of the stochastic particles to describe the distribution of these scalar variables. These stochastic particles can mix with each other and exchange the scalar variables to update the engine in-cylinder distributions of the gas species concentrations and soot moments during the combustion process. The number of the stochastic particles determines the precision of the physical and chemical predictions. Higher accuracy is achieved with more stochastic paritcles but at the expense of high computational cost. Usually 100 stochastic particles are found to be sufficient for engine simulations.

For diesel engine combustion simulation, a very important concern is the in-cylinder mixing intensity which significantly affects the mixture inhomogeneity inside the engine cylinder. This becomes even more important for cases where the soot formation process is modelled using a quadrature based method of moments such as the one used in this work. This is because the inappropriate mixing of soot moments would violate the realisability of the moment set, thus leading to unphysical soot particle size distributions. To avoid this problem, this work adopts the Curl's mixing model [58] to describe the turbulent mixing in which a pair of stochastic particles is chosen at a time and mixed to produce two new stochastic particles whose properties are the mean of the properties of the original ones. This mixing model has been proven to be able to maintain the realisability of the moment set [30].

\subsection{Soot model}

The soot model proposed by $[29,30]$ is adopted in this work to describe the soot formation and oxidation processes inside the diesel engines. The phys- 
ical and chemical processes considered to be important for the evolution of soot particles include: Inception, i.e. nucleation of the primary soot particles through dimerisation of pryene molecules, condensation, i.e. deposition of a pyrene molecule from the gas-phase to the surface of soot particles, surface growth, i.e. the addition of an acetylene molecule to the soot particle surface through a repeating cycle of H-radical abstraction and acetylene addition (HACA) mechanism [59], oxidation, i.e. removal of carbon atoms from the surface of soot particles due to reactions with hydroxyl radical and molecular oxygen, and coagulation is the modeled as the formation of larger soot particles due to the coalescent collision between soot particles. The detailed description of these processes is given in Appendix A.

Clearly, these soot particle processes may occur in a wide range of size classes, as a result an infinite number of soot population balance equations are needed to describe the soot PSD. This makes the simulation computationally extremely expensive, which is impractical for soot simulation in diesel engines. To reduce the computational cost, this work adopts the method of moments to treat the soot model. The moments of the soot PSD are defined as:

$$
M_{r}=\sum_{i=i_{0}}^{\infty} i^{r} N_{i}, \quad r=0,1, \cdots, \infty
$$

where $N_{i}$ is the number density of soot particles that contain $i$ carbon atoms. $M_{r}$ is the $r^{\text {th }}$-order moment. The lower-order moments usually have physical meanings. For example, $M_{0}$ is the total soot number and $M_{1}$ refers to the total soot size. Unfortunately the soot moment equations are unclosed. Especially, the evaluation of the soot oxidation source term requires the knowledge on the number of the smallest soot particles to account for 
the particle depletion term, which is challenging to MOM. To handle this problem, MPM is adopted to solve the soot moment equations. MPM has been shown to be able to treat the particle shrinkage problem (equivalent to oxidation) with high accuracy and at the same time retain the high computational efficiency. Refer to Appendix A for the detailed MPM formulation for the soot source terms.

\subsection{Particle size distribution reconstruction}

Starting from the moments obtained by MPM, the maximum entropy method is adopted as a post-process technique to reconstruct the particle size distribution. A brief description of the ME technique is given in this section. With ME, a positive distribution $\widetilde{N}_{K}$ is generated such that

$$
\sum_{i=i_{\min }}^{i_{\max }} i^{r} \widetilde{N}_{K}(i)=M_{r}, \quad r=0, \cdots, K,
$$

which eventually converge to the real PSD $N(i)$ as $K$ approaches infinity. The determination of $\widetilde{N}_{K}$ is achieved by finding the maximum entropy of $N(i)$ under the condition that the first $K+1$ moments of $\tilde{N}_{K}$ are equal to the moments of the real PSD. The entropy function of $N(i)$ is given as:

$$
S=-\sum_{i=i_{\min }}^{i_{\max }}(N(i) \ln N(i)-N(i))+\sum_{j=0}^{K} \lambda_{j}\left(\sum_{i=i_{\min }}^{i_{\max }} i^{j} N(i)-M_{j}\right) .
$$

where $\lambda_{j}(j=0, \cdots, K)$ are Lagrange multipliers. Taking the functional variation of Eq.(3) with respect to $(N(i))$ yields

$$
\frac{\delta S}{\delta N(i)}=0 \rightarrow N(i)=\widetilde{N}_{K}(i)=\exp \left(-\sum_{j=0}^{K} \lambda_{j} i^{j}\right)
$$


The key problem becomes to determine the Lagrange multipliers $\lambda_{j}$ so that $\widetilde{N}_{K}(i)$ can be used as an approximation of $N(i)$. Note that the first $K+1$ moments of $\widetilde{N}_{K}(i)$ are equal to the real moments:

$$
\sum_{i=i_{\min }}^{i_{\max }} i^{r} \widetilde{N}_{K}(i)=\sum_{i=i_{\min }}^{i_{\max }} i^{r} \exp \left(-\sum_{j=0}^{K} \lambda_{j} i^{j}\right)=M_{r}, \quad r=0, \cdots, K .
$$

Eq. 5 can be viewed as a nonlinear system of $K+1$ equations for the $K+1$ Lagrange multipliers. Unfortunately, the analytical solution of Eq. 5 rarely exists. For numerical purpose, the following convex potential is introduced:

$$
\Delta=\sum_{i=i_{\min }}^{i_{\max }}\left(\exp \left(-\sum_{j=0}^{K} \lambda_{j} i^{j}\right)-1\right)+\sum_{j=0}^{K} M_{j} \lambda_{j},
$$

One just needs to minimize the potential $\Delta$ as its stationary points are solutions to Eq. 5:

$$
\frac{\delta \Delta}{\delta \lambda_{r}}=0 \rightarrow \sum_{i=i_{\min }}^{i_{\max }} i^{r} \exp \left(-\sum_{j=0}^{K} \lambda_{j} i^{j}\right)=M_{r}, \quad r=0, \cdots, K .
$$

The Newton minimization procedure is adopted to determine these Langrange multipliers. Starting with some initial choices for $\lambda=\left(\lambda_{0}, \cdots, \lambda_{K}\right)^{\mathrm{T}}$, updated $\lambda$ by:

$$
\lambda=\lambda-\mathbf{H}^{-1}\left(\mathbf{M}-\langle\mathbf{I}\rangle_{\lambda}\right),
$$

where $\mathbf{H}$ is the Hessian matrix defined by $H_{m, n}=\frac{\partial^{2} \Delta}{\partial \lambda_{m} \partial \lambda_{n}}=\left\langle i^{m+n}\right\rangle_{\lambda}$ for $m, n=0, \cdots, K$, and $\langle\mathbf{I}\rangle_{\lambda}=\left(\left\langle i^{0}\right\rangle_{\lambda}, \cdots,\left\langle i^{K}\right\rangle_{\lambda}\right)^{\mathrm{T}}$ is the vector of approximated moments: 


$$
\left\langle i^{r}\right\rangle_{\lambda}=\sum_{i=i_{\min }}^{i_{\max }} i^{r} \exp \left(-\sum_{j=0}^{K} \lambda_{j} i^{j}\right) .
$$

The evaluation of $\left\langle i^{r}\right\rangle_{\lambda}$ can be done with the Gaussian quadrature approximations. Since the Gaussian quadrature points are computed for normalized densities at the interval $[0,1]$, a change of variabes is needed to re-locate the PSD from the interval $\left[i_{\min }, i_{\max }\right]$ to $[0,1]$ and the following transformation of moments has to be applied:

$$
\mu_{r}=\frac{M_{r}}{i_{\max }^{r} M_{0}}, \quad r=0, \cdots, K .
$$

This corresponds to the moments of normalized PSD located at $[0,1]$. After the PSD is reconstructed, a change of the variables back to the original interval is needed. It should be pointed out that the particle size interval $\left[i_{\min }, i_{\max }\right]$ is a key factor in determining the accuracy of ME. If it is set too narrow or too wide, unrealistic PSD might be generated. In MPM, the obtained weighted particles offer a proper way to approximate the interval. By fixing one weighted particle size $\alpha_{1}$ at $i_{\min }$, the largest weighted particle size $\alpha_{N_{\mathrm{p}}}$ can be used as a good reference of $i_{\max }$. In this work, $i_{\max }$ is determined by $i_{\max }=c \alpha_{N_{\mathrm{p}}}$. c is a user defined factor. It is found that satisfactory accuracy is always achieved by setting $c$ between 1 and 2. $c=1.5$ is adopted in this work. The detailed algorithm of the ME method is shown in Algorithm 1. 24-point Gaussian quadrature points are adopted for evaluations of Eq. 9. Since $\mathbf{H}$ is symmetric positive definite, the classical Choleski decomposition is used to solve the linear system in Eq.(8). 
Input: A $K \times 1$ vector $\mathbf{M}$ with elements are the moments $\widetilde{M}_{r}(r=0, \cdots, K-1)$; the minimum particle size $i_{\min }$ and the maximum particle size $i_{\max }$; The absolute tolerance $\varepsilon$.

Output: The reconstructed $\operatorname{PSD} \tilde{N}(i), i=i_{\min }, \cdots, i_{\max }$.

Initialize the 24-point Gaussian quadratures:

$\mathrm{x}=[-0.064056893,0.064056893,-0.191118867,0.191118867,-0.31504268,0.31504268,-0.433793508,0.433793508$, $-0.545421471,0.545421471,-0.648093652,0.648093652,-0.740124192,0.740124192,-0.820001986,0.820001986$, $-0.886415527,0.886415527,-0.938274552,0.938274552,-0.974728556,0.974728556,-0.99518722,0.99518722]$

$\mathrm{w}=[0.127938195,0.127938195,0.125837456,0.125837456,0.121670473,0.121670473$, $0.115505668,0.115505668,0.10744427,0.10744427,0.097618652,0.097618652$, $0.086190162,0.086190162,0.073346481,0.073346481,0.059298585,0.059298585$, $0.044277439,0.044277439,0.028531389,0.028531389,0.01234123,0.01234123]$

Create a $K \times 1$ vector $\mu$ with elements are:

$$
\mu_{r}=\frac{\widetilde{M}_{r}}{i_{\max }^{r} \widetilde{M}_{0}} .
$$

This corresponds to the moments of normalized PSD at the interval $[0,1]$. for $N=1$ to $K-1$ do

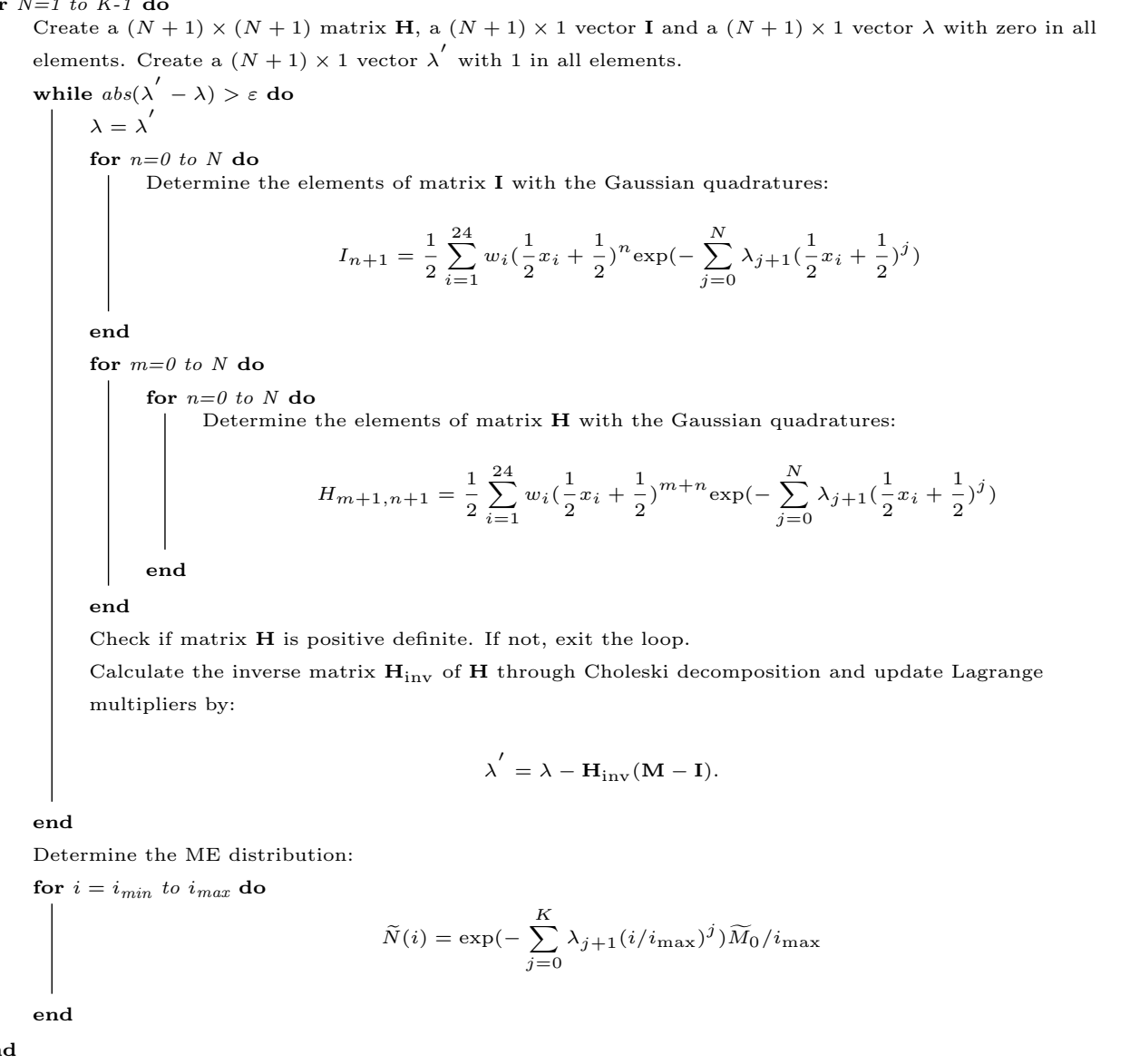

\section{Algorithm 1: Maximum entropy algorithm.}




\section{MPM-ME evaluation}

Before applying the joint MPM-ME approach to the simulation of soot formation in diesel engines, the performances of this approach for the treatment of PBEs and PSD reconstruction are evaluated first on MatLab. A simplified particle population balance model is adopted where the contributions of gas-phase species are neglected, and constant inception rates and collision kernels are applied. The PBE describing such particle system is given as:

$$
\frac{\mathrm{d} n_{i}}{\mathrm{~d} t}=r(i)+g(i)+w^{\mathrm{g}}(i)+w^{\mathrm{ox}}(i)
$$

Note that lower case letters are used to avoid confusions with the soot model described in Appendix A. The specific particle source terms are:

$$
\begin{aligned}
r(i) & =I_{0}, \\
g(i) & =\frac{1}{2} \sum_{j=1}^{i-1} \beta_{j, i-j} n_{j} n_{i-j}-\sum_{j=1}^{\infty} \beta_{i, j} n_{i} n_{j}, \\
w^{\mathrm{g}}(i) & =k_{\mathrm{g}}(i-1) n_{i-1}-k_{\mathrm{g}}(i) n_{i}, \\
w^{\mathrm{ox}}(i) & =k_{\mathrm{ox}}(i+1) n_{i+1}-k_{\mathrm{ox}}(i) n_{i},
\end{aligned}
$$

where $I_{0}$ represents the inception rate. The surface reaction kernels $k_{\mathrm{g}}(i)$ and $k_{\mathrm{ox}}(i)$ are modeled to be propertional to the particle size: $k_{\mathrm{g}}(i)=k_{\mathrm{g}} i$ and $k_{\mathrm{ox}}(i)=k_{\mathrm{ox}} i$, where $k_{\mathrm{g}}$ and $k_{\mathrm{ox}}$ are constants. The coagulation kernel under the continuum regime is used:

$$
\beta_{i, j}=K_{c}\left(\frac{1}{i^{1 / 3}}+\frac{1}{j^{1 / 3}}+K_{c}^{\prime}\left(\frac{1}{i^{2 / 3}}+\frac{1}{j^{2 / 3}}\right)\right)\left(i^{1 / 3}+j^{1 / 3}\right),
$$


where $K_{c}$ and $K_{c}^{\prime}$ are assumed to be constants. Two test cases are adopted where the oxidation kernel is varied to simulate relatively strong (Case 1) and weak (Case 2) oxidation:

Case 1 Inception rate $I_{0}=500 \mathrm{~s}^{-1}$, growth kernel $k_{\mathrm{g}}=2 \mathrm{~s}^{-1}$, oxidation kernel $k_{\mathrm{ox}}=10 \mathrm{~s}^{-1}$, coagulation kernels $K_{c}=10^{-6} \mathrm{~s}^{-1}$ and $K_{c}^{\prime}=2$.

Case 2 Inception rate $I_{0}=500 \mathrm{~s}^{-1}$, growth kernel $k_{\mathrm{g}}=2 \mathrm{~s}^{-1}$, oxidation kernel $k_{\mathrm{ox}}=0.5 \mathrm{~s}^{-1}$, coagulation kernels $K_{c}=10^{-6} \mathrm{~s}^{-1}$ and $K_{c}^{\prime}=2$.

Simulations are performed with a log-normal distribution supplied as the initial condition:

$$
n(i, t=0)=100 \exp \left(-(\log (i)-\log (40))^{2} / 0.2\right) .
$$

In MPM, four weighted particle size classes $\left(N_{\mathrm{p}}=4\right)$ are adopted to treat the PBEs. In total $7\left(2 N_{\mathrm{p}}-1\right)$ moments are calculated. The performance of MPM is assessed by comparing the model solution with the direct numerical simulations. Figure 1 shows the moments obtained for case 1. For this case, a large oxidation kernel is adopted to simulate a oxidation-dominant condition. Therefore, both the total particle number as represented by $M_{0}$ and total particle size represented by $M_{1}$ exhibit a gradual decrease. The moments obtained using MPM match the exact solution perfectly, suggesting the high accuracy of MPM in solving the particle PBEs.

Given the moments obtained by MPM, the particle PSDs at different simulation time are reconstructed using the ME method with 7 Lagrange multipliers. Figure 2 compares the reconstructed PSDs with the original ones. Since oxidation is dominating the particle process, a shift of the PSD towards the smallest size is observed. The number of the smallest particles exhibits an 

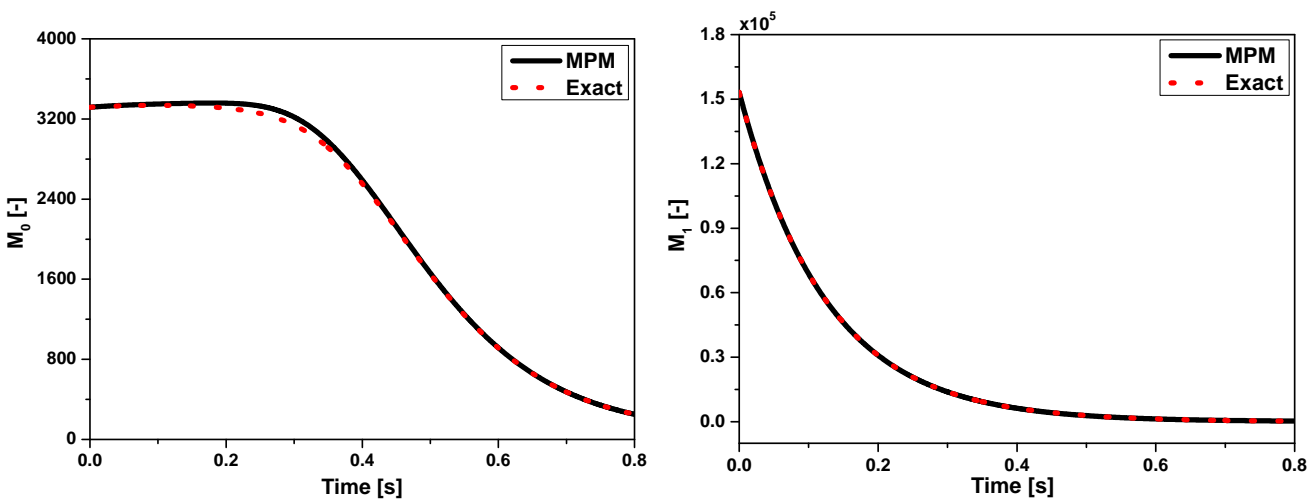

Figure 1: Comparison of the zeroth moment $M_{0}$ (left panel) and the first moment $M_{1}$ (right panel) between MPM and the direct numerical simulation for case 1.

increase at the beginning resulting from the transformation from the large particles, then starts to decrease due to their own oxidation. In general, the reconstructed PSDs match well to the exact solutions, indicating the high performance of the ME method adopted in this work.

Case 2 simulates a weak oxidation process. The moments obtained for this case with MPM and the direct numerical simulation are shown in Fig. 3. An increase in the total particle number and size is observed. Again, MPM generates almost the same moments with the direct numerical solution. This further indicates the high accuracy of MPM.

The original and reconstructed PSDs at different simulation time for case 2 are shown in Fig. 4. A shift of the PSD towards the large particle size is observed. At the same time, the peak becomes wider and lower. Due to the persistent inception at the smallest particle size and formation of large particles through growth, the PSD exihibits a bimodal distribution. In general, the PSDs reconstructed by ME match satisfactorily well to the exact 


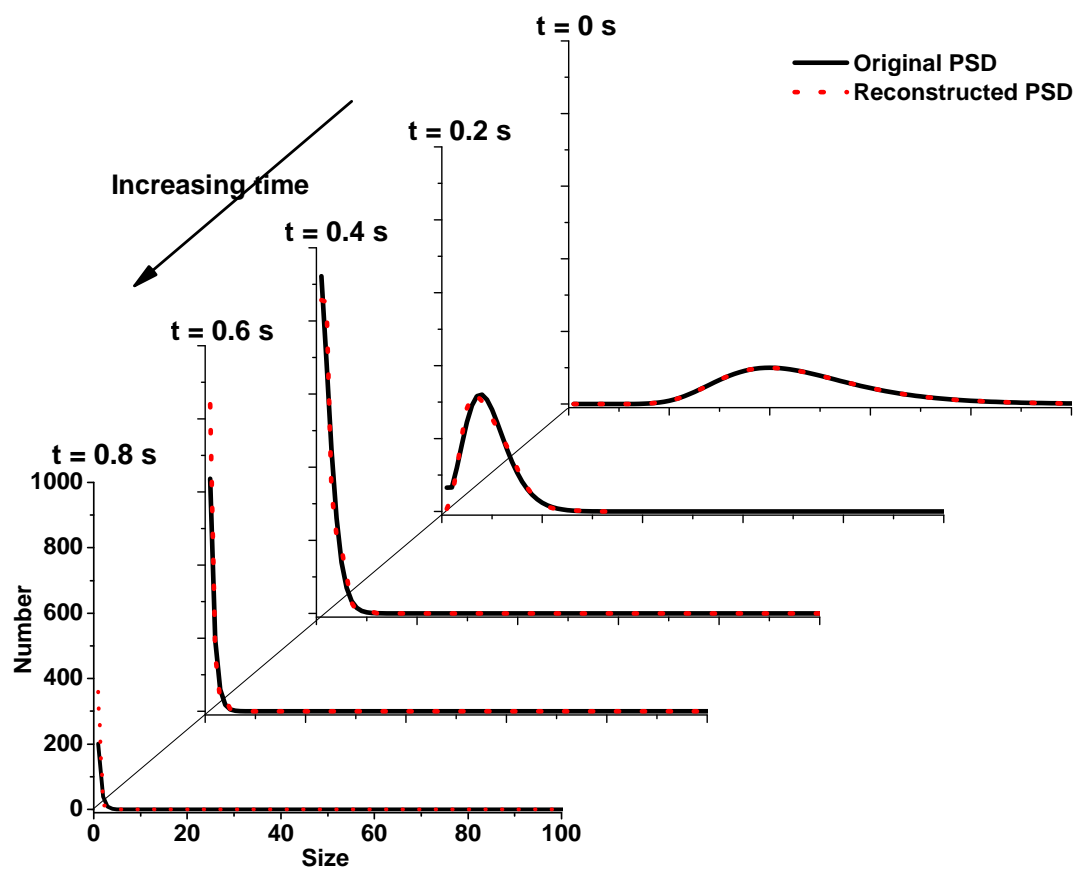

Figure 2: Comparison between the original PSD and the reconstructed PSD obtained by ME for case 1.
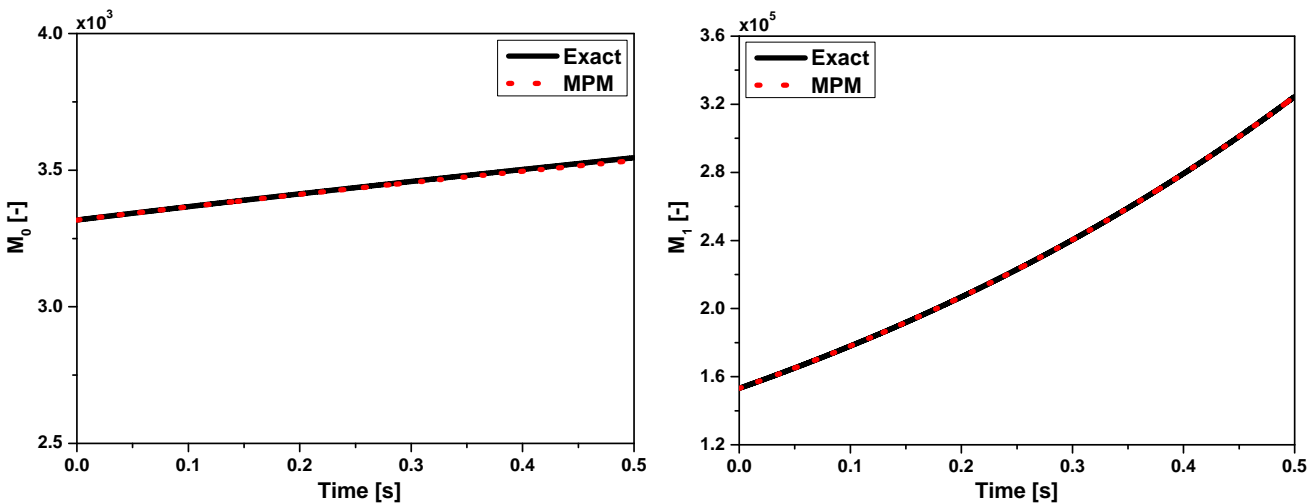

Figure 3: Comparison of the zeroth moment $M_{0}$ (left panel) and the first moment $M_{1}$ (right panel) between MPM and the direct numerical simulation for case 2. 
solution.

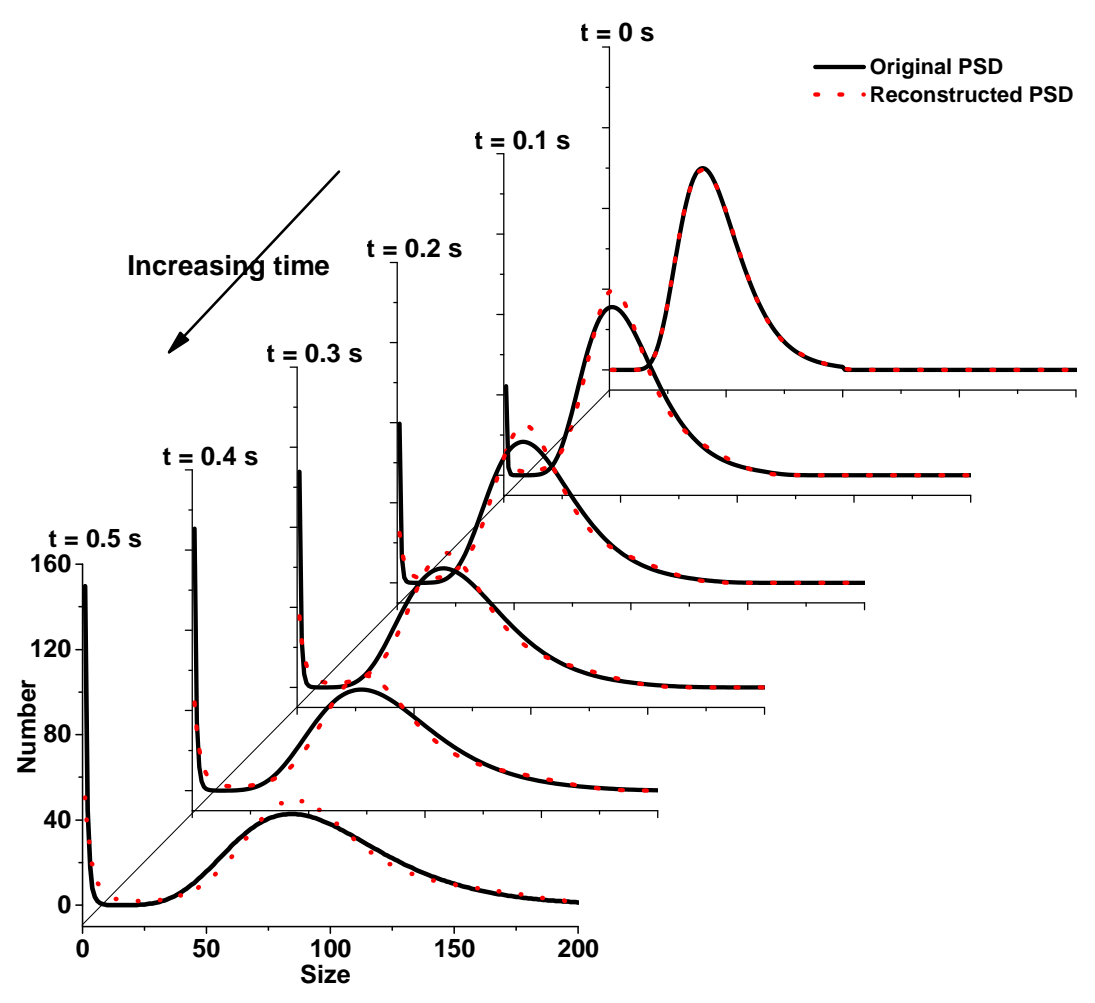

Figure 4: Comparison between the original PSD and the reconstructed PSD obtained by ME for case 2.

In summary, the proposed joint MPM-ME approach in this work is able to solve the particle PBEs and reconstruct the PSD with satisfactory accuracy.

\section{Diesel engine simulation}

In this section, the joint MPM-ME approach is implemented into the SRM engine code to simulate the combustion process in a single-cylinder directinjection diesel engine from [60]. The specifications of the engine are summarized in Table 1. The engine was running at a constant speed of $1500 \mathrm{rpm}$ 
with three different loads. The operating conditions are given in Table 2. In the experiment, the engine in-cylinder pressure was measured using a Kistler 6215C piezoelectric sensor. An exhaust flow dilution channel was built on the diesel engine experimental bench and a low pressure impactor was adopted to collect the emitted soot particles. Refer to [60] for the detailed experimental setup. Simulations were performed with a time step of $0.2 \mathrm{CAD}$ and 100 stochastic particles. A detailed mechanism for Primary Reference Fuel (PRF) containing 208 species and 1002 reactions is employed [35]. The soot model described in section 2.2 is used to simulate the soot formation process. In MPM, three weighted particle size classes $\left(N_{\mathrm{p}}=3\right)$ were used to treat the soot PBEs. In total 5 soot moments were solved for.

Figure 5 compares the predicted in-cylinder pressure and heat release rates with the experimental measurements for case 1. Satisfactory match between the simulation results and experimental data has been achieved. The ignition timing and combustion duration have been well captured, suggesting that the combustion process inside the diesel engine has been successfully modeled. To evaluate the additional CPU costs induced by including the soot model in the diesel engine simulations, the cases that only consider gas-phase chemistry and those with both gas-phase chemistry and soot particle dynamics are simulated. The CPU costs are compared and summarized in Fig. 6. Note that for all cases the CPU time is normalized by the data obtained where only gas-phase chemistry is considered. As can be seen the inclusion of the soot model does not contribute much computational cost owing to the high efficiency of MPM. The CPU time is increased by around only $20 \%$. This is very promising concerning the fact that traditionally the treatment of 
Table 1: Diesel engine specification.

\begin{tabular}{ll}
\hline Engine type & Single-cylinder, four-stroke, water-cooled \\
Bore & $105 \mathrm{~mm}$ \\
Stroke & $115 \mathrm{~mm}$ \\
Compression ratio & $18: 1$ \\
Displacement & $996 \mathrm{~cm}^{3}$ \\
Rated power & $12.1 \mathrm{~kW}$ \\
Rated speed & $2300 \mathrm{r} / \mathrm{min}$ \\
Start of injection & $25^{\circ} \mathrm{CA} \mathrm{BTDC}$ \\
Injection duration & $28^{\circ} \mathrm{CA}$ \\
Spray angle & $154^{\circ} \mathrm{CA}$ \\
Nozzle diameter & $0.28 \mathrm{~mm}$ \\
Number of nozzle & 5 \\
Injection pressure & $30 \mathrm{MPa}$ \\
\hline
\end{tabular}

Table 2: Engine operating conditions.

\begin{tabular}{llll}
\hline & Case 1 & Case 2 & Case 3 \\
\hline Engine speed (r/min) & 1500 & 1500 & 1500 \\
Torque $(\mathrm{N} \mathrm{m})$ & 40 & 30 & 20 \\
\hline
\end{tabular}


the soot model using the stochastic method usually makes the diesel engine simulation prohibitively expensive.

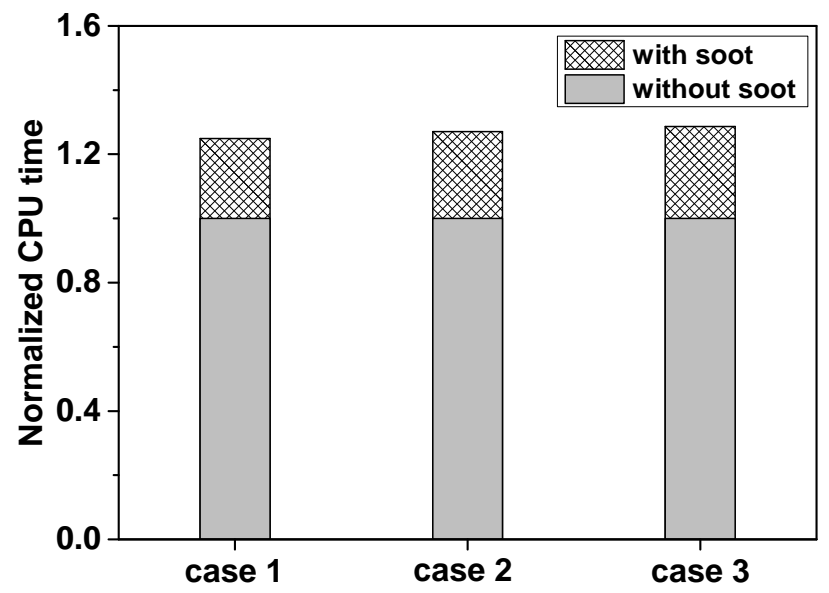

Figure 6: Evaluation of the additional CPU costs induced by including the soot model in the diesel engine simulation. For all the cases, the CPU time is normalized by the data obtained without considering the soot model. 
In Fig. 7, simulation and experimental results of the engine-out soot mass for all the cases are summarized. Both the experimental and simulation results have been normalized by the data for case 1 . As can be seen the soot model correctly captures the trend of the soot emissions. With the engine load increasing, more diesel fuels are consumed and correspondingly the emitted soot mass is increased. However, the predicted soot emissions exhibit some discrepancies with the experimental results, which is a consequence of the inappropriate soot model parameters. The parameters describing the soot formation and oxidation rates are derived from a limited number of experimental or theoretical studies. There is a lack of concensus within the literatures in terms of these parameters. Therefore, the soot model parameters usually have to be properly calibrated in order to achieve better agreement between the simulation results and experimental data. However, it should be noted that the purpose of this paper is not to calibrate the model parameters to fit the experimental data but to propose a new numerical approach through which a comprehensive study on the soot formation and oxidation processes inside diesel engines could be achieved. Next the detailed evolution of soot particle population inside the diesel engine obtained by the proposed MPM-ME approach for case 1 is presented.

Figure 8 shows the modeled rates of $M_{0}$ and $M_{1}$ for different particle processes for case 1 . Note that the coagulation rate for $M_{1}$, condensation rate and growth rate for $M_{0}$ are zero, hence not shown in the figures. The coagulation rate for $M_{0}$ and oxidation rates for $M_{0}$ and $M_{1}$ are negative, here we take their absolute values for illustration. As can be seen, all the moment rates exhibit a sharp increase soon after ignition. Due to the strong fluctuations 


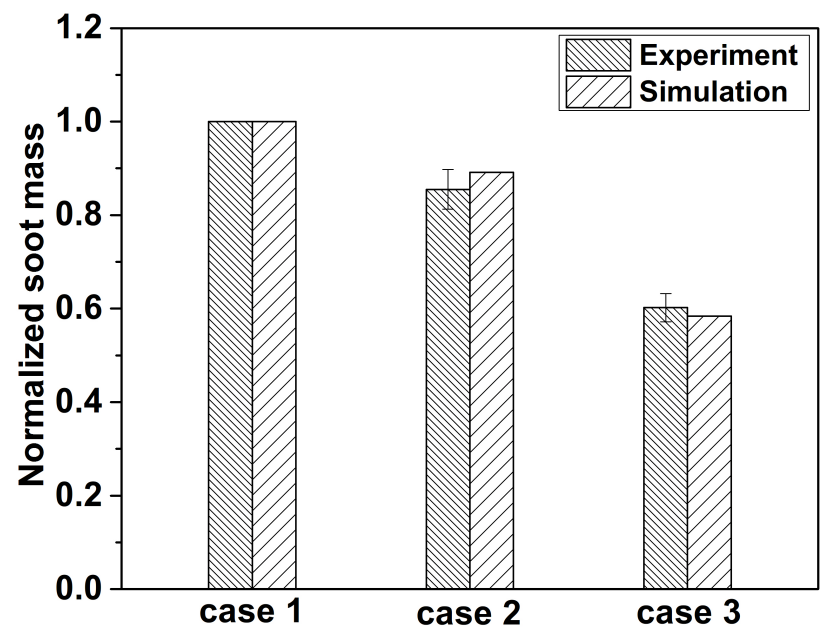

Figure 7: Comparison of soot mass between experiments and simulations. All experimental data are normalized by the experimental value measured for case 1 condition, and all model data are normalized by the predicted value calculated for case 1 condition.

in turbulent mixing, the moment rates exhibit quite a bit of noise during the main combustion process. As the combustion strength weakens, the noises become eliminating and these moment rates show a gradual decrease. The oxidation rate via $\mathrm{OH}$ is found to be much larger than that via $\mathrm{O}_{2}$, suggesting that the soot oxidation is mainly dominated by external burning process while internal burning plays a minor role.

Figure 9 shows the predicted molar concentrations of acetylene and pyrene as a function of crank angle for case 1. Pyrene is considered as the soot precursor in the current soot model and acetylene plays a major role in soot growth process via the HACA mechanism. The evolution of these two species therefore has a significant effect on soot formation. As can be seen from Fig. 9 these two species exhibit a similar evolution trend with the heat release rate 

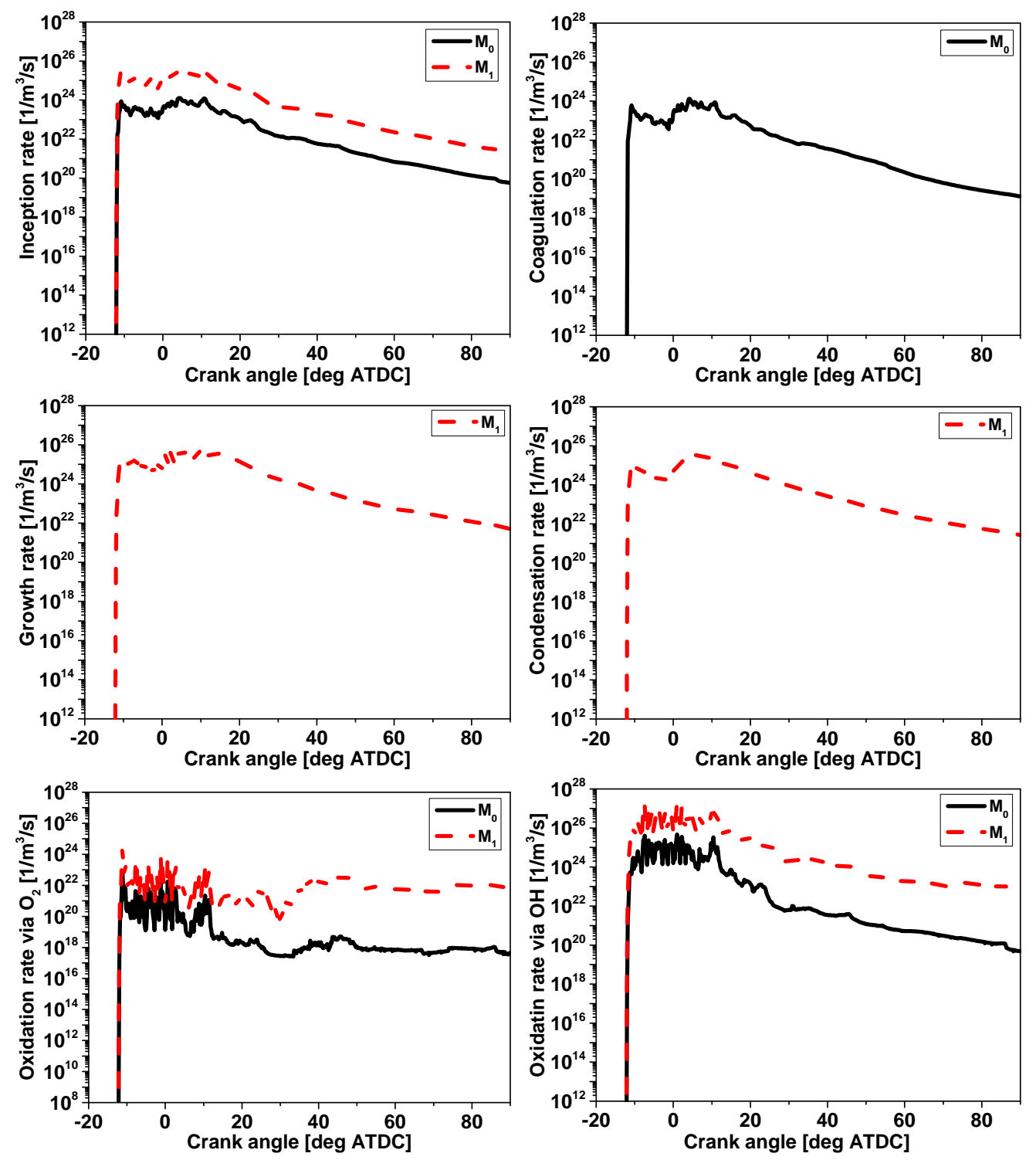

Figure 8: Modeled soot moment rates as a function of crank angle for case 1.

as shown in Fig. 5. Soon after ignition, the molar concentrations of both species show a sharp increase as a resut of the pyrolysis and oxidation of diesel fuel. Due to the rapid chemical reactions during the main combustion stage, 
the molar concentrations of both species exhibit a lot of noises. Then starting from around 30 deg ATDC, the combustion strength becomes weakened and a decrease of the molar concentrations of both species is observed.

Figure 10 shows the predicted soot number and mass as a function of crank angle for case 1 . At the beginning, inception dominates the soot formation process, leading to a fast build up of soot particles inside the engine cylinder. Then oxidation starts to gain strength and the total soot number shows a decrease trend during the late combustion stage. By contrast the total soot mass exhibits a steady increase due to the accumulation of particles at the large sizes which can be revealed by the following reconstructed soot PSD.
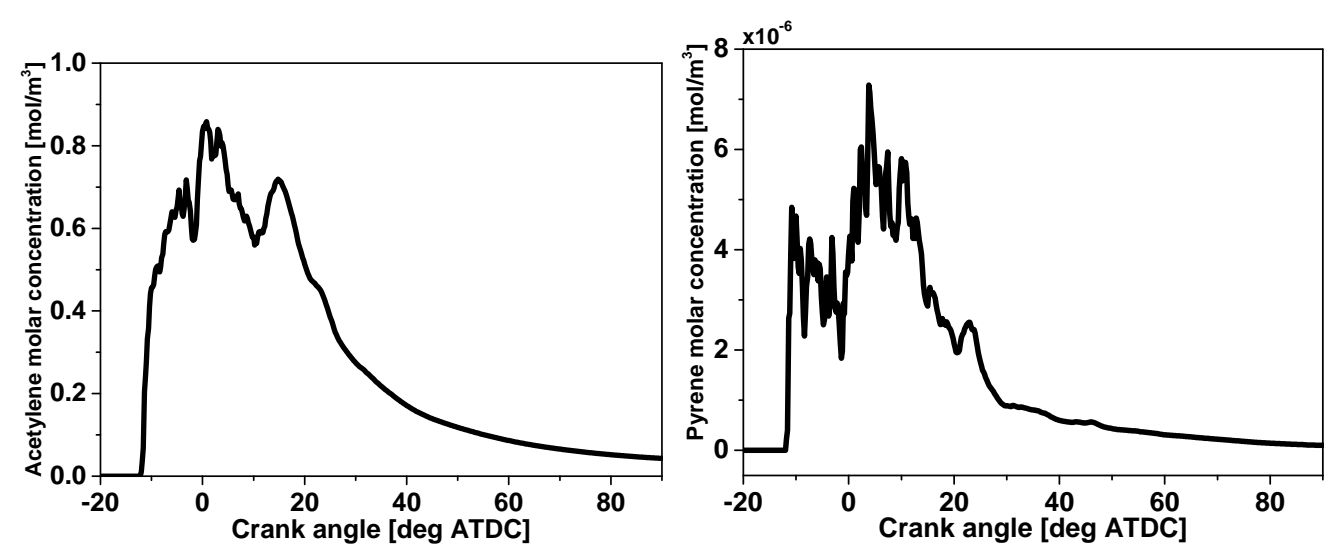

Figure 9: Predicted molar concentrations of acetylene and pyrene as a function of crank angle for case 1.

As already mentioned, the soot PSD $N_{i}$ used in the soot model is based on the carbon atom number $i$, which can be as large as $10^{8}$ during the combustion process in diesel engines. If applying this to Eq. 10, numerical errors may occur and the reconstructed PSD is not realistic. In order to avoid this 

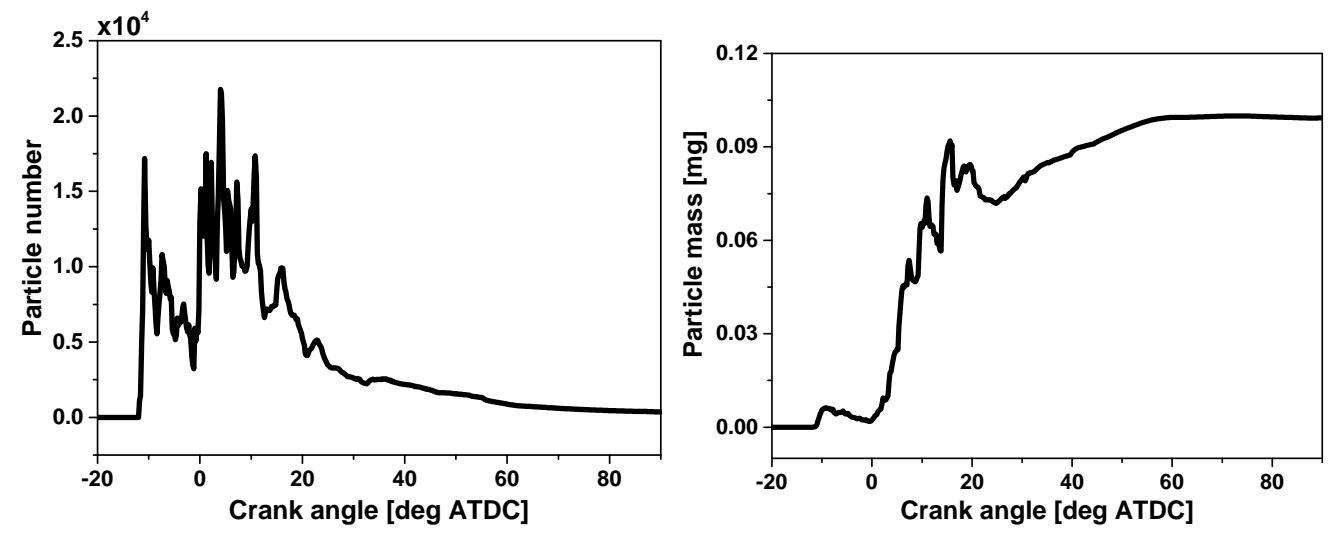

Figure 10: Modeled soot number and mass as a function of crank angle for case 1 .

problem, a transformation from the carbon atom number to the particle collision diameter is implemented:

$$
d=\left(\frac{6 m_{1}}{\pi \rho_{s}}\right)^{1 / 3} i^{1 / 3}
$$

where $d$ is the collision diameter of particles that contain $i$ carbon atoms. $m_{1}$ is the mass of a single carbon atom and $\rho_{s}$ is the density of soot particles. The soot collision diameter $d$ is usually in the size range of [1 nm, $1000 \mathrm{~nm}$ ] which can be used by ME with much lower numerical errors. Correspondingly, the following collision diameter based moments $M_{r}^{*}$ are used as the input moments for the ME method:

$$
M_{r}^{*}=\left(\frac{6 m_{1}}{\pi \rho_{s}}\right)^{r / 3} M_{r / 3},
$$

where the fractional-order moments $M_{r / 3}$ are determined by using the polynomial interpolation technique based on the integer moments $M_{r}$.

Figure. 11 shows the reconstructed soot PSD as a function of crank angle 
for case 1. As can be seen at -12 deg ATDC where ignition just occurs, the particle process is dominated by inception. The soot PSD exhibits a unimodal distribution located at the smallest size. As inception continues, an increase in the total soot particle number is observed. Furthermore, particles are formed at larger sizes as growth and coagulation start to gain strength. Under the combined effect of persistent inception, growth, condensation and coagulation, the soot PSD starts to exhibit a bimodal distribution. During the late combustion stage, the oxidation starts to gain strength and the total soot particle number begins to decrease gradually. Furthermore, an accumulation of particles at large sizes is observed under the persistent effect of growth and coagulation.

Due to the high rotation speed and harsh combustion condition in diesel engines, it is difficult to extract the soot particles from the engine cylinder at fixed crank angle. Therefore very limited experimental information on the soot PSD exists for comparison. However, it should be noted that the main purpose of this work is not to calibrate the soot model parameters to fit the experimental data, but to propose a new approach for simulating soot formation and oxidation in diesel engines. The developed MPM-ME method is robust, accurate and computationally cheap. At the same time it is able to provide a detailed analysis on the soot particle dynamics in diesel engines. 

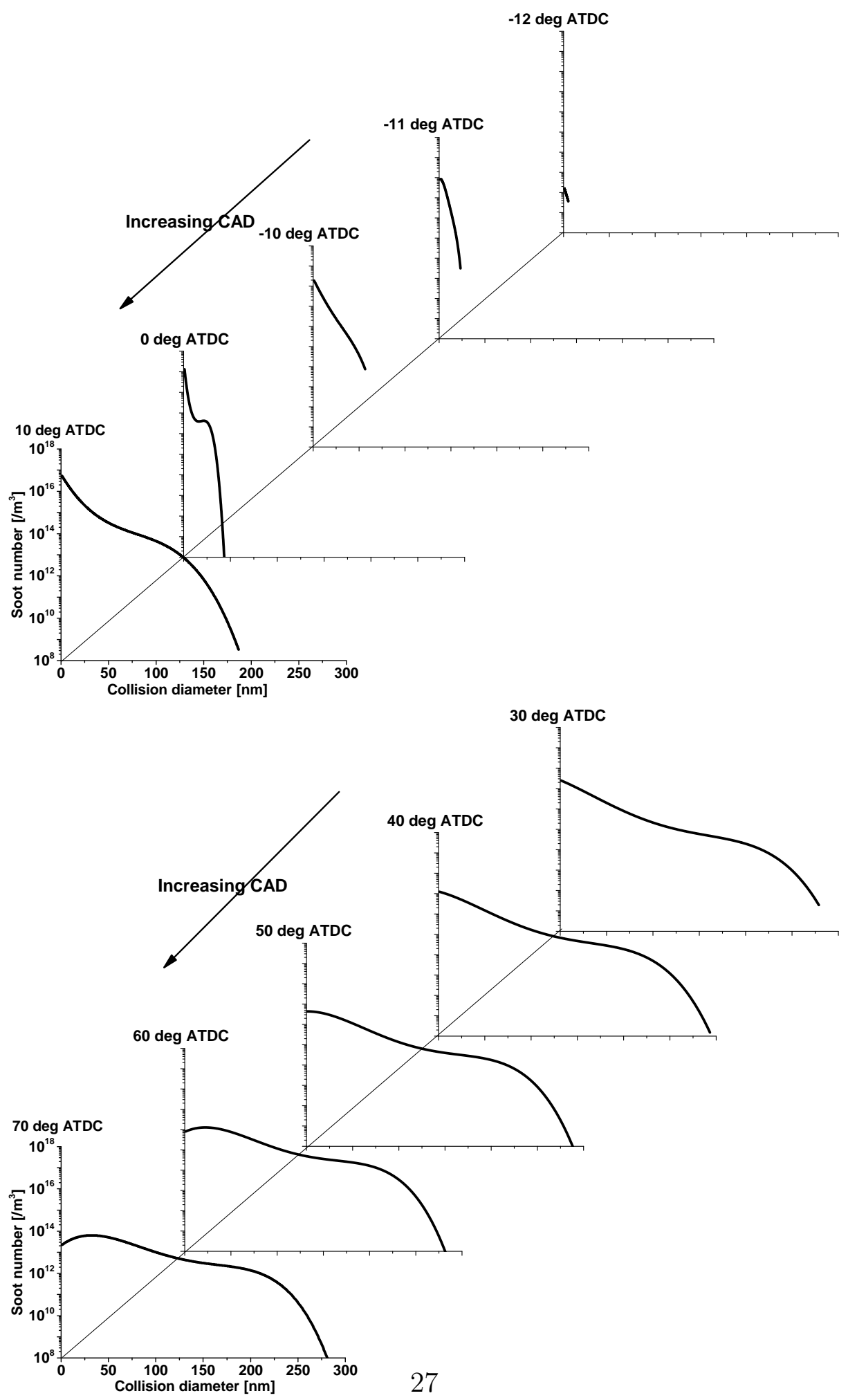

Figure 11: Reconstructed soot PSD as a function of crank angle for case 1. The top panel refers to the main combustion stage and the bottom panel refers to the late combustion stage. 


\section{Conclusion}

This work presents a new approach, a joint moment projection method and maximum entropy reconstruction, for simulating soot formation and oxidation in diesel engines. With this approach, the moment projection method is adopted to solve the soot population balance equations including inception, coagulation, condensation, surface growth and oxidation. The obtained moments are then supplied to the maximum entropy approach, which is adopted as a post-processing technique, to reconstruct the soot particle size distribution. The particle size range, which is a key factor in affecting the accuracy of maximum entropy approach, is determined based on the weighted particles generated in the moment projection method. The performance of this approach is first evaluated by solving a set of simplified population balance equations on MatLab. Both cases where oxidation is dominant and weak are simulated. The moment projection method has shown to be very accurate in solving the population balance equations. The obtained moments are almost the same with the direct numerical simulations. The reconstructed particle size distribution by the maximum entropy approach match satisfactorily well with the exact solutions for both cases considered. The bimodal distribution of the particles due to persistent inception and growth are successfully reproduced. Afterwards, the joint moment projection method and maximum entropy approach is implemented into a probability density function based stochastic reactor model engine code to describe the soot formation and oxidation processes inside a single-cylinder direct-injection diesel engine. Three test cases are applied where the diesel engine is operated under different engine loads. Results suggest that the inclusion of the soot model induces little 
CPU cost owing to the high efficiency of the moment projection method. For all the cases considered, the model is able to correctly capture the emission trend of soot. The soot moment rates for all particle processes together with the evolutions of the total soot number and soot size are obtained. The soot particle size distributions at different crank angles are reconstructed, which enables a physical interpretation of the soot particle dynamics during the combustion process. The proposed joint moment projection method and maximum entropy approach in this work is accurate, easy to implement and computationally cheap. It offers a comprehensive study on the soot formation and oxidation processes in diesel engines. Complete information of the soot particle size distribution can be provided, resolving a long-standing limitation of the method of moments.

\title{
Acknowledgement
}

This research is supported by the National Research Foundation, Prime Minister's Office, Singapore under its CREATE programme.

\section{Nomenclature}

\author{
Upper-case Roman \\ $C_{s}$ Transformation factor \\ $G$ Source term due to coagulation \\ H Hessian matrix
}


$I_{0} \quad$ Inception rate

I Vector with components are a function of reconstructed particle size distribution

$K \quad$ Number

$K_{c}, K_{c}^{\prime}$ Coagulation kernels

$M$ Moment

M Matrix with components which are a function of moments

$N$ Number

$R$ Source term due to inception

$S$ Entropy function of particle size distribution

$W \quad$ Source term due to surface reactions

$$
\begin{aligned}
\text { Lower-case Roman } \\
d \quad \text { Diameter } \\
g \quad \text { Source term due to coagulation } \\
i, j \quad \text { particle size } \\
k_{\mathrm{g}} \quad \text { Growth kernel } \\
k_{\mathrm{ox}} \quad \text { Oxidation kernel } \\
k, i, j, r \quad \text { Indices } \\
k_{\mathrm{g}} \quad \text { Growth kernel } \\
k_{\mathrm{ox}} \quad \text { Oxidation kernel } \\
r \quad \text { Source term due to inception } \\
m \quad \text { Mass }
\end{aligned}
$$




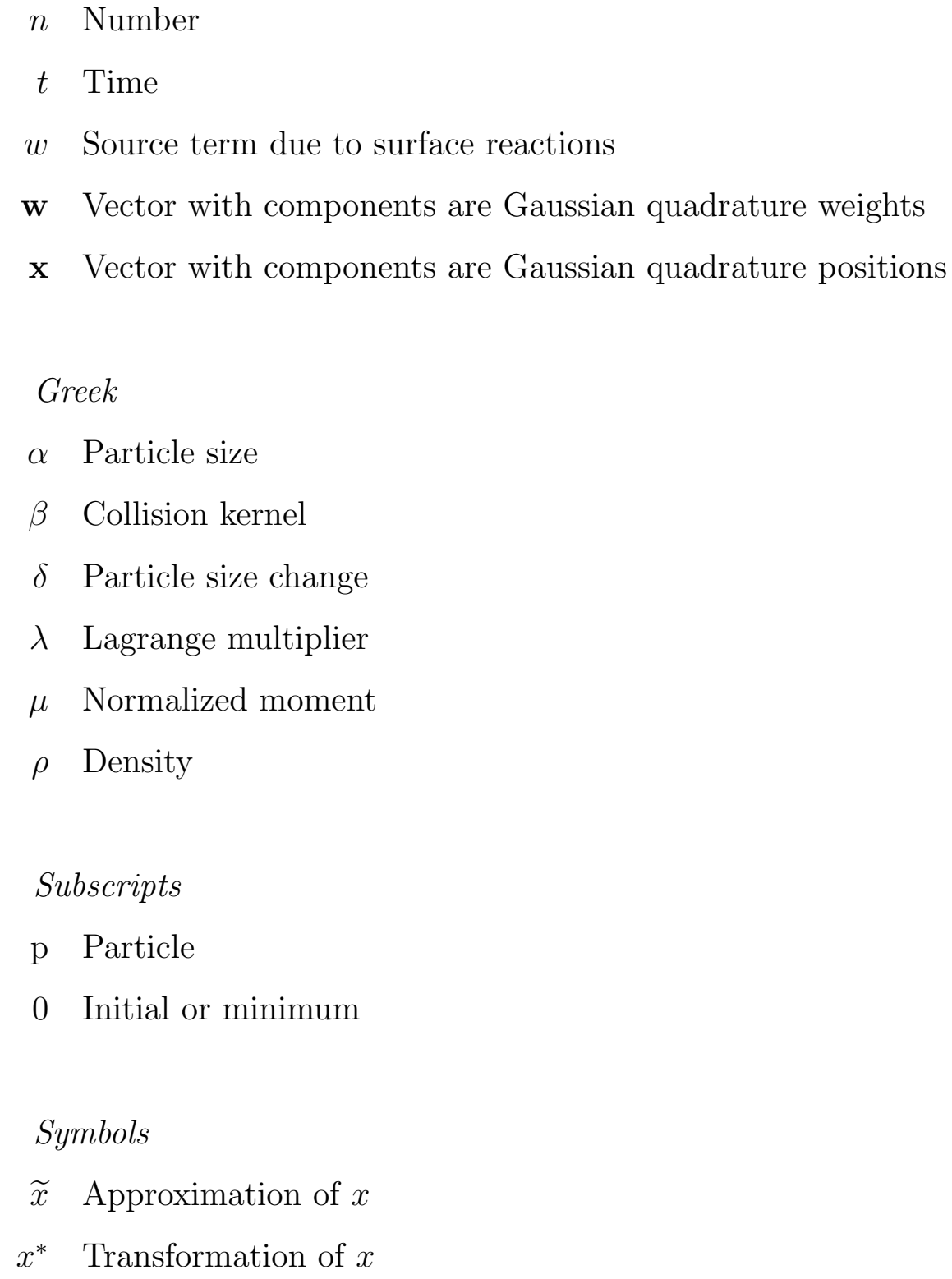




\author{
Abbreviations \\ DICI Direct injection compression ignition \\ DISI Direct injection spark ignition \\ EQMOM Extended quadrature method of moments \\ FCMOM Finite-size domain complete set of trial functions method of mo- \\ ments \\ HACA $\mathrm{H}$-radical abstraction and acetylene addition \\ HCCI Homogeneous charge compression ignition \\ PAH Polycyclic aromatic hydrocarbon \\ PBE Population balance equation \\ PDF Probability density function \\ PSD Particle size distribution \\ ME Maximum entropy \\ MOM Method of moments \\ MPM Moment projection method \\ DSA Direct simulation algorithm \\ SRM Stochastic reactor model
}




\section{Appendix A. MPM closure for soot formation and oxidation source terms}

In this work, the soot is modeled as a population of spherical particles consisting of merely carbon atoms. Within each stochastic particle, the evolution of the soot particle population is governed by:

$$
\frac{\mathrm{d} N_{i}}{\mathrm{~d} t}=R(i)+G(i)+W^{\mathrm{cond}}(i)+W^{\mathrm{g}}(i)+W^{\mathrm{ox}}(i),
$$

where $N_{i}$ refers to the number density of soot particles of the size $i$. The terms on the right-hand side of the above equation represent, respectively, inception, coagulation, condensation, surface growth and oxidation.

Inception: The primary soot particles are assumed to be formed when two PAH molecules collide and stick with each other. The sticking probability of the PAHs is determined with a collision efficiency model. In this work, it is assumed that the primary soot particle is formed after two pyrene molecules stick together after a collision with the source term given as:

$$
R\left(i_{0}\right)=\frac{1}{2} \beta_{\text {in }} N_{\text {pyrene }}^{2}
$$

where $N_{\text {pyrene }}$ is the pyrene number density and $\beta_{\text {in }}$ is the collision rate of pyrene molecules. $i_{0}$ refers to size of the primary soot particles.

Coagulation: Once the primary soot particles are formed, the population of the particles can evolve through collision and sticking among these particles. The coagulation process is modeled using the Smoluchowski equation [61]:

$$
G(i)=\frac{1}{2} \sum_{j=i_{0}}^{i-1} \beta_{j, i-j} N_{j} N_{i-j}-\sum_{j=i_{0}}^{\infty} \beta_{i, j} N_{i} N_{j} .
$$


$\beta_{i, j}$ is the collision kernel describing the rate of successful collisions between two soot particles. The first term on the right-hand side describes the creation of particles due to collisions between all combinations of particles with sizes that sum to $i$. It contains a factor of $1 / 2$ to avoid double counting. The second term describes the destruction of particles due to collisions between particles of size $i$ and any other size $j$.

Condensation: The soot particle may grow in size due to the deposition of the PAH molecule on the particle surface following a collision between the two partners. The collision rate is determined as per coagulation except that one of the collision partners is PAH which is modeled as pyrene in this work:

$$
W^{\text {cond }}(i)=\beta_{i-\delta, \delta} N_{\text {pyrene }} N_{i-\delta}-\beta_{i, \delta} N_{\text {pyrene }} N_{i},
$$

$\delta$ refers to the change of the particle size and is modeled as 16 since pyrene is adopted as the condensation species.

Surface growth: Surface growth results from a repeating cycle of H-radical abstraction and acetylene addition followed by the formation of a new aromatic ring on the soot particle surface (HACA mechanism) [59]. The reaction rate depends on the soot surface radical sites and is assumed to be proportional to the surface area:

$$
W^{\mathrm{g}}(i)=k_{\mathrm{g}}\left(s_{i-\delta} N_{i-\delta}-s_{i} N_{i}\right) .
$$

$k_{\mathrm{g}}$ represents the growth kernel and $s_{i}$ is the particle surface area. $\delta$ is set as 2 since acetylene is adopted as the growth species.

Oxidation: The carbon atoms at the surface of soot particles can be removed due to the surface reactions with molecular oxygen and hydroxyl radicals. 
When oxidized, the large soot particles are turned into the small ones and the smallest are decomposed into gas phase species, leading to a decrease of the total soot particle number. The oxidation source term is given as:

$$
W^{\mathrm{ox}}(i)=k_{\mathrm{ox}}\left(s_{i-\delta} N_{i-\delta}-s_{i} N_{i}\right) .
$$

$k_{\text {ox }}$ refers to the oxidation kernel. $\delta$ is set as -2 and -1 , respectively, for oxidation via $\mathrm{O}_{2}$ and $\mathrm{OH}$.

As already mentioned, an efficient approach to solve these soot PBEs is to transform them into moment equations and solve for just the first few lowerorder moments so that the computational cost can be kept to the minimum. The moment of the soot PSD is defined as:

$$
M_{r}=\sum_{i=i_{0}}^{\infty} i^{r} N_{i}, \quad r=0,1, \cdots, \infty
$$

where $M_{r}$ is the $r^{\text {th }}$-order moment. The lower-order moments usually have physical meanings. For example, $M_{0}$ is the total soot number and $M_{1}$ refers to the total soot size. The soot moment equations can be obtained by applying Eq. A.7 to Eq. A.1:

$$
\frac{d M_{r}}{d t}=R_{r}+G_{r}+W_{r}^{\mathrm{cond}}+W_{r}^{\mathrm{g}}+W_{r}^{\mathrm{ox}},
$$

where the moment source terms are: 


$$
\begin{aligned}
& R_{r}=\frac{1}{2} i_{0}^{r} \beta_{\text {pyrene }} N_{\text {pyrene }}^{2}, \\
& G_{r}= \begin{cases}-\frac{1}{2} \sum_{i=i_{0}}^{\infty} \sum_{j=i_{0}}^{\infty} \beta_{i, j} N_{i} N_{j}, & r=0 \\
0, & r=1 \\
\frac{1}{2} \sum_{i=i_{0}}^{\infty} \sum_{j=i_{0}}^{\infty} \sum_{k=1}^{r-1}\left(\begin{array}{l}
r \\
k
\end{array}\right) i^{k} j^{r-k} \beta_{i, j} N_{i} N_{j}, & r>1\end{cases} \\
& W_{r}^{\text {cond }}= \begin{cases}0, & r=0 \\
\sum_{i=i_{0}}^{\infty} \sum_{k=0}^{r-1}\left(\begin{array}{l}
r \\
k
\end{array}\right) i^{k} \delta^{r-k} \beta_{i, \delta} N_{\text {pyrene }} N_{i}, & r>1\end{cases} \\
& W_{r}^{\mathrm{g}}= \begin{cases}0, & r=0 \\
k_{\mathrm{g}} \sum_{i=i_{0}}^{\infty} \sum_{k=0}^{r-1}\left(\begin{array}{ll}
r \\
k
\end{array} i^{k} j^{r-k} s_{i} N_{i},\right. & r>1\end{cases} \\
& W_{r}^{\mathrm{ox}}= \begin{cases}-k_{\mathrm{ox}} \sum_{i=i_{0}}^{i_{0}-1-\delta} s_{i} N_{i}, & r=0 \\
k_{\mathrm{ox}} \sum_{i=i_{0}}^{\infty} \sum_{k=0}^{r-1}\left(\begin{array}{l}
r \\
k
\end{array} i^{k} j^{r-k} s_{i} N_{i}-k_{\mathrm{ox}} \sum_{i=i_{0}}^{i_{0}-1-\delta}(i+\delta)^{r} s_{i} N_{i} .\right. & r>1\end{cases}
\end{aligned}
$$

The evaluation of these source terms depends on the kernels adopted. In this work, the kernels and rates related with the soot formation and surface reactions are taken from $[29,30,62]$. The application of these realistic kernel functions leads to the presence of fractional-order moments which are not directly solved for and have to be properly estimated to close the moment 
equations. Another closure problem arises from the oxidation moment source term. As seen in Eq. A.13, the numbers of the smallest soot particles are present which refer to the soot depletion process due to oxidation. The evaluation of this soot depletion term is challenging to moment methods since the detailed information of the soot PSD is lost as it has been transformed into moments.

In this work, the moment projection method is adopted to close these moment source terms. The basic idea behind MPM is to approximate the soot PSD using a set of weighted particles that are located into a finite number of particle size classes. The moments of the approximated PSD is expressed as:

$$
\widetilde{M}_{r}=\alpha_{1}^{r} \widetilde{N}_{\alpha_{1}}+\sum_{j=2}^{N_{\mathrm{p}}} \alpha_{j}^{r} \widetilde{N}_{\alpha_{j}}, \quad r=0, \ldots, 2 N_{\mathrm{p}}-2,
$$

where $\alpha_{j}$ is the approximated weighted particle size, $\widetilde{N}_{\alpha_{j}}$ is the weight, and $N_{\mathrm{p}}$ is the number of weighted particle sizes adopted. These weighted particles have to meet the requirement that the corresponding empirical moments are equal to the moments from the true PSD:

$$
\widetilde{M}_{r}=M_{r}, \quad r=0, \ldots, 2 N_{\mathrm{p}}-2 .
$$

In order to evaluate the soot depletion term due to oxidation, one of the weighted particle sizes is fixed at the smallest soot size, $\alpha_{1}=i_{0}$, so that the corresponding weight $\widetilde{N}_{\alpha_{1}}$ can be used to approximate the number of the smallest soot particles. The moment source terms within the framework of MPM is given as: 


$$
\frac{\mathrm{d} \widetilde{M}_{r}}{\mathrm{~d} t}=R_{r}(\widetilde{M})+G_{r}(\widetilde{M})+W_{r}^{\mathrm{cond}}(\widetilde{M})+W_{r}^{\mathrm{g}}(\widetilde{M})+W_{r}^{\mathrm{ox}}(\widetilde{M}, \widetilde{N})
$$

where the specific moment source terms are:

$$
\begin{aligned}
& R_{r}(\widetilde{M})=\frac{1}{2} i_{0}^{r} \beta_{\text {pyrene }} N_{\text {pyrene }}^{2} \\
& G_{r}(\widetilde{M})= \begin{cases}-\frac{1}{2} \sum_{i=1}^{N_{\mathrm{p}}} \sum_{j=1}^{N_{\mathrm{p}}} \beta_{\alpha_{i}, \alpha_{j}} \widetilde{N}_{\alpha_{i}} \widetilde{N}_{\alpha_{j}}, & r=0 \\
0, & r=1 \\
\frac{1}{2} \sum_{i=1}^{N_{\mathrm{p}}} \sum_{j=1}^{N_{\mathrm{p}}} \sum_{k=1}^{r-1}\left(\begin{array}{l}
r \\
k
\end{array}\right) \alpha_{i}^{k} \alpha_{j}^{r-k} \beta_{\alpha_{i}, \alpha_{j}} \widetilde{N}_{\alpha_{i}} \widetilde{N}_{\alpha_{j}}, & r>1\end{cases} \\
& W_{r}^{\text {cond }}(\widetilde{M})= \begin{cases}0, & r=0 \\
\sum_{j=1}^{N_{\mathrm{p}}} \sum_{k=0}^{r-1}\left(\begin{array}{l}
r \\
k
\end{array}\right) \alpha_{j}^{k} \delta^{r-k} \beta_{\alpha_{j}, \delta} N_{\text {pyrene }} \widetilde{N}_{\alpha_{j}}, & r>1\end{cases} \\
& W_{r}^{\mathrm{g}}(\widetilde{M})= \begin{cases}0, & r=0 \\
k_{\mathrm{g}} \sum_{j=1}^{N_{\mathrm{p}}} \sum_{k=0}^{r-1}\left(\begin{array}{l}
r \\
k
\end{array}\right) \alpha_{j}^{k} \delta^{r-k} s_{\alpha_{j}} \widetilde{N}_{\alpha_{j}}, & r>1\end{cases} \\
& W_{r}^{\mathrm{ox}}(\widetilde{M}, \widetilde{N})= \begin{cases}-k_{\mathrm{ox}} s_{\alpha_{1}} \tilde{N}_{\alpha_{1}}, & r=0 \\
k_{\mathrm{Ox}} \sum_{j=1}^{N_{\mathrm{p}}} \sum_{k=0}^{r-1}\left(\begin{array}{l}
r \\
k
\end{array}\right) \alpha_{j}^{k} \delta^{r-k} s_{\alpha_{j}} \widetilde{N}_{\alpha_{j}}-k_{\mathrm{Ox}}\left(\alpha_{1}+\delta\right)^{r} s_{\alpha_{1}} \widetilde{N}_{\alpha_{1}} \cdot & r>1\end{cases}
\end{aligned}
$$

The determination of the weighted particles is achieved by using a Blumstein 
and Wheeler algorithm [63] based on the obtained moments. See $[50,51]$ for the detailed algorithm of MPM. 
[1] M. Bolla, D. Farrace, Y. M. Wright, K. Boulouchos, Modelling of soot formation in a heavy-duty diesel engine with conditional moment closure, Fuel 117 (2014) 309-325. doi:10.1016/j.fuel.2013.09.041.

[2] J. Boulanger, F. Liu, W. S. Neill, G. J. Smallwood, An improved soot formation model for 3D diesel engine simulations, J. Eng. Gas Turb. Power. 129 (2007) 877-884. doi:10.1115/1.2718234.

[3] A. J. Torregrosa, A. Broatch, A. García, L. F. Mónico, Sensitivity of combustion noise and NOx and soot emissions to pilot injection in PCCI Diesel engines, Appl. Energy 104 (2013) 149-157. doi:10.1016/j.apenergy.2012.11.040.

[4] D. Agarwal, S. K. Singh, A. K. Agarwal, Effect of Exhaust Gas Recirculation (EGR) on performance, emissions, deposits and durability of a constant speed compression ignition engine, Appl. Energy 88 (2011) 2900-2907. doi:10.1016/j.apenergy.2011.01.066.

[5] C. J. Mueller, C. W. Nilsen, D. J. Ruth, R. K. Gehmlich, L. M. Pickett, S. A. Skeen, Ducted fuel injection: A new approach for lowering soot emissions from direct-injection engines, Appl. Energy 204 (2017) 206220. doi:10.1016/j.apenergy.2017.07.001.

[6] K. Donaldson, L. Tran, L. A. Jimenez, R. Duffin, D. E. Newby, N. Mills, W. MacNee, V. Stone, Combustion-derived nanoparticles: A review of their toxicology following inhalation exposure, Part. Fibre Toxicol. 2 (2005) 1-14. doi:10.1186/1743-8977-2-10.

[7] P. Eastwood, Particulate emissions from vehicles, John Wiley, 2008. 
[8] J. Liu, F. Yang, H. Wang, M. Ouyang, S. Hao, Effects of pilot fuel quantity on the emissions characteristics of a $\mathrm{CNG} /$ diesel dual fuel engine with optimized pilot injection timing, Appl. Energy 110 (2013) 201-206. doi:10.1016/j.apenergy.2013.03.024.

[9] C. D. Rakopoulos, A. M. Dimaratos, E. G. Giakoumis, D. C. Rakopoulos, Study of turbocharged diesel engine operation, pollutant emissions and combustion noise radiation during starting with bio-diesel or n-butanol diesel fuel blends, Appl. Energy 88 (2011) 3905-3916. doi:10.1016/j.apenergy.2011.03.051.

[10] D. B. Hulwan, S. V. Joshi, Performance, emission and combustion characteristic of a multicylinder DI diesel engine running on dieselethanolbiodiesel blends of high ethanol content, Appl. Energy 88 (2011) 50425055. doi:10.1016/j.apenergy.2011.07.008.

[11] K. M. Pang, N. Karvounis, J. H. Walther, J. Schramm, Numerical investigation of soot formation and oxidation processes under large two-stroke marine diesel engine-like conditions using integrated CFD-chemical kinetics, Appl. Energy 169 (2016) 874-887. doi:10.1016/j.apenergy.2016.02.081.

[12] T. Li, T. Yin, B. Wang, Anatomy of the cooled EGR effects on soot emission reduction in boosted spark-ignited direct-injection engines, Appl. Energy 190 (2017) 43-56. doi:10.1016/j.apenergy.2016.12.105.

[13] A. Kazakov, D. Foster, Modeling of soot formation during DI diesel 
combustion using multistep phenomenological model, SAE Paper 107 (1998) 1016-1028. doi:10.4271/982463.

[14] P. Belardini, C. Bertoli, A. Ciajolo, A. D'Anna, N. DelGiacomo, Three Dimensional Calculations of DI Diesel Engine Combustion and Comparison whit In Cylinder Sampling Valve Data, SAE Paper 101 (1992) 1899-1908. doi:10.4271/922225.

[15] F. Zhao, W. Yang, W. Yu, H. Li, Y. Y. Sim, T. Liu, K. L. Tay, Numerical study of soot particles from low temperature combustion of engine fueled with diesel fuel and unsaturation biodiesel fuels, Appl. Energy 211 (2018) 187-193. doi:10.1016/j.apenergy.2017.11.056.

[16] H. Hiroyasu, T. Kadota, Models for Combustion and Formation of Nitric Oxide and Soot in Direct Injection Diesel Engines, SAE Technical Paper 85 (1976) 513-526. doi:10.4271/760129.

[17] H. Hiroyasu, K. Nishida, Simplified three-dimensional modeling of mixture formation and combustion in D. I. diesel engine, SAE Technical Paper 98 (1989) 276-293. doi:10.4271/890269.

[18] M. Patterson, S. C. Kong, G. Hampson, R. D. Reitz, Modeling the Effects of Fuel Injection Characteristics on Diesel Engine Soot and NOx Emissions, SAE Paper 103 (1994) 836-852. doi:10.4271/940523.

[19] A. R. Wadhwa, V. Gopalakrishnan, J. Abraham, A Mixture Fraction Averaged Approach to Modeling NO and Soot in Diesel Engines, SAE Technical Paper 110 (2001) 955-970. doi:10.4271/2001-01-1005. 
[20] J. Nagle, R. F. Strickland-Constable, Oxidation of carbon between 1000$2000{ }^{\circ} \mathrm{C}$, Proc. of the Fifth Carbon Conference (1962) 154-164.

[21] V. Fraioli, C. Beatrice, M. Lazzaro, Soot particle size modelling in 3D simulations of diesel engine combustion, Combust. Theor. Model. 15 (2011) 863-892. doi:10.1080/13647830.2011.578662.

[22] S. Hong, M. S. Wooldridge, H. G. Im, D. N. Assanis, E. Kurtz, Modeling of diesel combustion, soot and NO emissions based on a modified eddy dissipation concept, Combust. Sci. and Tech. 180 (2008) 14211448. doi:10.1080/00102200802119340.

[23] M. Pasternak, F. Mauss, C. Perlman, H. Lehtiniemi, Aspects of 0D and 3D modeling of soot formation for diesel engines, Combust. Sci. Technol. 186 (2014) 1517-1535. doi:10.1080/00102202.2014.935213.

[24] S. Wu, W. Yang, H. Xu, Y. Jiang, Investigation of soot aggregate formation and oxidation in compression ignition engines with a pseudo bi-variate soot model, Appl. Energy 253 (2019) 1-12. doi:10.1016/j.apenergy.2019.113609.

[25] S. Wu, D. Zhou, W. Yang, Implementation of an efficient method of moments for treatment of soot formation and oxidation processes in three-dimensional engine simulations, Appl. Energy 254 (2019) 1-12. doi:10.1016/j.apenergy.2019.113661.

[26] J. E, T. Liu, W. Yang, Y. Deng, J. Gong, A skeletal mechanism modeling on soot emission characteristics for biodiesel surrogates with varying 
fatty acid methyl esters proportion, Appl. Energy 181 (2016) 322-331. doi:10.1016/j.apenergy.2016.08.090.

[27] A. Trubetskaya, P. A. Jensen, A. D. Jensen, A. D. G. Llamas, K. Umeki, D. Gardini, J. Kling, R. B. Bates, P. Glarborg, Effects of several types of biomass fuels on the yield, nanostructure and reactivity of soot from fast pyrolysis at high temperatures, Appl. Energy 171 (2016) 468-482. doi:10.1016/j.apenergy.2016.02.127.

[28] A. Trubetskaya, A. Brown, G. A. Tompsett, M. T. Timko, J. Kling, M. Broström, M. L. Andersen, K. Umeki, Characterization and reactivity of soot from fast pyrolysis of lignocellulosic compounds and monolignols, Appl. Energy 212 (2018) 1489-1500. doi:10.1016/j.apenergy.2017.12.068.

[29] J. Appel, H. Bockhorn, M. Frenklach, Kinetic modeling of soot formation with detailed chemistry and physics: Laminar premixed flames of C2 hydrocarbons, Combust. Flame. 121(1-2) (2000) 122-136. doi:10.1016/S0010-2180(99)00135-2.

[30] M. Balthasar, F. Mauss, A. Knobel, M. Kraft, Detailed Modeling of Soot Formation in a Partially Stirred Plug Flow Reactor, Combust. Flame. 128 (2002) 395-409. doi:10.1016/S0010-2180(01)00344-3.

[31] M. E. Mueller, G. Blanquart, H. Pitsch, A joint volume-surface model of soot aggregation with the method of moments, Proc. Combust. Inst. 32 (2009) 785-792. doi:10.1016/j.proci.2008.06.207. 
[32] D. L. Marchisio, A. A. Barresi, Investigation of soot formation in turbulent flames with a pseudo-bivariate population balance model, Chem. Eng. Sci. 64 (2009) 294-303. doi:10.1016/j.ces.2008.10.020.

[33] D. Ramkrishna, Population Balances: Theory and Applications to Particulate Systems in Engineering, Academic Press, New York, 2000.

[34] J. Etheridge, S. Mosbach, M. Kraft, H. Wu, N. Collings, Modelling soot formation in a DISI engine, Proc. Combust. Inst. 33 (2011) 3159-3167. doi:10.1016/j.proci.2010.07.039.

[35] S. Mosbach, M. S. Celnik, A. Raj, M. Kraft, H. R. Zhang, S. Kubo, K. O. Kim, Towards a detailed soot model for internal combustion engines, Combust. Flame. 156 (2009) 1156-1165. doi:10.1016/j.combustflame.2009.01.003.

[36] B. Wang, S. Mosbach, S. Schmutzhard, S. Shuai, Y. Huang, M. Kraft, Modelling soot formation from wall films in a gasoline direct injection engine using a detailed population balance model, Appl. Energy 163 (2016) 154-166. doi:10.1016/j.apenergy.2015.11.011.

[37] K. F. Lee, R. I. A. Patterson, W. Wagner, M. Kraft, Stochastic weighted particle methods for population balance equations with coagulation, fragmentation and spatial inhomogeneity, J. Comput. Phys. 303 (2015) 1-18. doi:10.1016/j.jcp.2015.09.031.

[38] R. I. A. Patterson, W. Wagner, M. Kraft, Stochastic weighted particle methods for population balance equations, J. Comput. Phys. 230 (19) (2011) 7456-7472. doi:10.1016/j.jcp.2011.06.011. 
[39] M. Balthasar, M. Kraft, A stochastic approach to calculate the particle size distribution function of soot particles in laminar premixed flames, Combust. Flame 133 (3) (2003) 289-298. doi:10.1016/S00102180(03)00003-8.

[40] E. K. Y. Yapp, R. I. A. Patterson, J. Akroyd, S. Mosbach, E. M. Adkins, J. H. Miller, M. Kraft, Numerical simulation and parametric sensitivity study of optical band gap in a laminar coflow ethylene diffusion flame, Combust. Flame 167 (2016) 320-334. doi:10.1016/j.combustflame.2016.01.033.

[41] M. Frenklach, Method of moments with interpolative closure, Chem. Eng. Sci. 57 (12) (2002) 2229-2239. doi:10.1016/S0009-2509(02)001136 .

[42] D. L. Marchisio, R. D. Vigil, R. O. Fox, Quadrature method of moments for aggregation-breakage processes, J. Colloid Interface Sci. 258 (2003) 322-334. doi:10.1016/S0021-9797(02)00054-1.

[43] D. L. Marchisio, J. T. Pikturna, R. O. Fox, R. D. Vigil, A. A. Barresi, Quadrature method of moments for population-balance equations, AIChE. J. 49 (2003) 1266-1276. doi:10.1002/aic.690490517.

[44] D. L. Marchisio, R. O. Fox, Solution of population balance equations using the direct quadrature method of moments, J. Aerosol Sci. 36 (2005) 43-73. doi:10.1016/j.jaerosci.2004.07.009.

[45] S. Wu, C. Lindberg, J. Akroyd, W. Yang, M. Kraft, Bivariate extension of the moment projection method for the particle popula- 
tion balance dynamics, Comput. Chem. Eng. 124 (2019) 206-227. doi:10.1016/j.compchemeng.2018.12.011.

[46] M. E. Mueller, G. Blanquart, H. Pitsch, Modeling the oxidation-induced fragmentation of soot aggregates in laminar flames, Proc. Combust. Inst. 33 (1) (2011) 667-674. doi:10.1016/j.proci.2010.06.036.

[47] M. Massot, F. Laurent, D. Kah, C. S. D., A robust moment method for evaluation of the disappearance rate of evaporating sprays, Siam J. Appl. Math. 70 (2010) 3203-3234. doi:10.1137/080740027.

[48] A. Wick, T. T. Nguyen, F. Laurent, R. O. Fox, H. Pitsch, Modeling soot oxidation with the Extended Quadrature Method of Moments, Proc. Combust. Inst. 36 (2017) 789-797. doi:10.1016/j.proci.2016.08.004.

[49] R. B. Diemer, J. H. Olson, A moment methodology for coagulation and breakage problems: part 2 - moment models and distribution reconstruction, Chem. Eng. Sci. 57 (12) (2002) 2211-2228. doi:10.1016/S00092509(02)00112-4.

[50] S. Wu, E. K. Y. Yapp, J. Akroyd, S. Mosbach, R. Xu, W. Yang, M. Kraft, A moment projection method for population balance dynamics with a shrinkage term, J. Comput. Phys. 330 (2017) 960-980. doi:10.1016/j.jcp.2016.10.030.

[51] S. Wu, E. K. Y. Yapp, J. Akroyd, S. Mosbach, R. Xu, W. Yang, M. Kraft, Extension of moment projection method to the fragmentation process, J. Comput. Phys. 335 (2017) 516-534. doi:10.1016/j.jcp.2017.01.045. 
[52] E. Madadi-Kandjani, A. Passalacqua, An extended quadrature-based moment method with log-normal kernel density functions, Chem. Eng. Sci. 131 (2015) 323-339. doi:10.1016/j.ces.2015.04.005.

[53] M. Strumendo, H. Arastoopour, Solution of PBE by MOM in finite size domains, Chem. Eng. Sci. 63 (2008) 2624-2640. doi:10.1016/j.ces.2008.02.010.

[54] C. Yuan, F. Laurent, R. O. Fox, An extended quadrature method of moments for population balance equations, J. Aerosol Sci. 51 (2012) 1-23. doi:10.1016/j.jaerosci.2012.04.003.

[55] L. R. Mead, N. Papanicolaou, Maximum entropy in the problem of moments, J. Math. Phys. 25 (1984) 2404-2417. doi:10.1063/1.526446.

[56] S. Wu, W. Yang, Comparisons of methods for reconstructing particle size distribution from its moments, Fuel 252 (2019) 325-338. doi:10.1016/j.fuel.2019.04.124.

[57] A. Smallbone, A. Bhave, A. Coble, S. Mosbach, M. Kraft, R. McDavid, Identifying optimal operating points in terms of engineering constraints and regulated emissions in modern diesel engines, SAE Technical Paper 2011-01-1388. doi:http://dx.doi.org/10.4271/2011-01-1388.

[58] R. L. Curl, Dispersed phase mixing: I. theory and effects in simple reactors, A.I.Ch.E. J. 9 (1963) 175-181. doi:10.1002/aic.690090207.

[59] M. Frenklach, H. Wang, Detailed modeling of soot particle nucleation and growth, Proc. Combust. Inst 23 (1991) 1559-1566. doi:10.1016/S0010-2180(99)00135-2. 
[60] H. Ju, Study on formation laws and size distribution of soot in the diesel engines, Huazhong University of Science and Technology, 2011.

[61] M. Von Smoluchowski, Investigation of a mathematical theory on the coagulation of colloidal suspensions, Z. Phys. Chem. (1917) 92-129.

[62] H. Wang, M. Frenklach, A detailed kinetic modeling study of aromatics formation in laminar premixed acetylene and ethylene flames, Combust. Flame 110 (1997) 173-221. doi:10.1016/S0010-2180(97)00068-0.

[63] C. Blumstein, J. C. Wheeler, Modified-moments method: applications to harmonic solids, Phys. Rev. B 8 (1973) 1764-1776. doi:10.1103/PhysRevB.8.1764. 